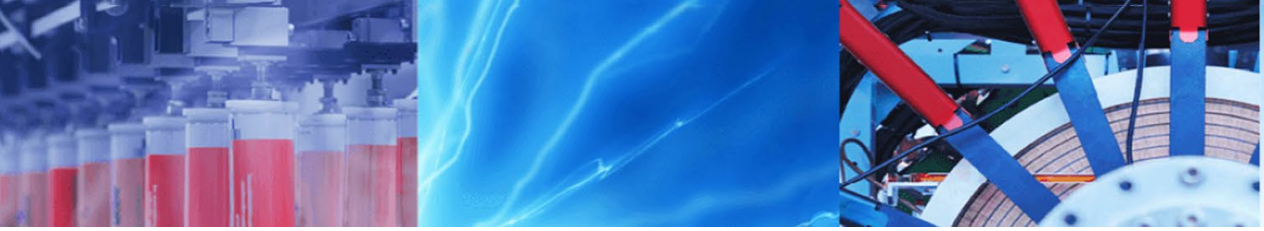

Research Article

\title{
Hesperidin: synthesis and characterization of bioflavonoid complex
}

\author{
Iwona Binkowska' ${ }^{1}$
}

Received: 16 December 2019 / Accepted: 12 February 2020 / Published online: 18 February 2020

(c) The Author(s) $2020 \quad$ OPEN

\begin{abstract}
Flavonoids are widely recognized for their beneficial effects in the cosmetic industry, possessing many biological activities, such as antioxidant, anti-inflammatory and antimicrobial properties. The study presented an efficient and simple solution to improve the preparations of antioxidant complexes based on hesperidin. Obtained products are characterized by thermogravimetric, spectrophotometric method, electron scanning microscopy, color analysis and zeta potential. Lightness value $\left(L^{*}\right)$ of hesperidin-silica complexes was found to be inversely correlated with the antioxidant activity values.
\end{abstract}

Keywords Flavonoid $\cdot$ Antioxidant $\cdot$ Hesperidin $\cdot$ Spectrophotometric $\cdot$ Zeta potential

\section{Introduction}

Flavonoids are a class of compounds founds in plant cells. These polyphenolic compounds are generally nontoxic and belong to the natural pigments family. In food, they are responsible for taste and color. Existing research confirm that a number of positive health effects of flavonoids primarily results from their antioxidant activity. Flavonoids are responsible: for transition metal chelating such as copper and iron, so that prevent free radicals, interrupting a series of reaction of free radical in enzymatic and non-enzymatic lipid peroxidation [1-4]. Among of this large class of interesting compounds, hesperidin is a bioflavonoid found in citrus fruits. Structurally, hesperidin is a $\beta$-7-rutinoside of hesperetin consists of an aglycone, hesperetin and a disaccharide, rutinose. Anticancer and cancer chemopreventive properties of hesperidin and hesperetin are reported in literature [5]. It was interesting that these results are confirmed by in vitro and in vivo experiments. Many papers described the beneficial effects of the supplementation with hesperidin and hesperetin on the cardiovascular system include antihypertensive, anticoagulant, cardioprotective effects against oxidative stress [6]. Current strategies to improve these properties include formation of derivatives of natural products. It was also demonstrated that the silver nanoparticles synthesized using hesperidin showed antibacterial effects and cytotoxicity [7]. In literature, the binding properties of hesperetin and its copper complex with calf thymus DNA were investigated. Tan et al. found that complex of flavonoid with $\mathrm{Cu}$ (II) may be a potential metal-based anticancer drug [8]. Cao et al. described preparation and characterization of complex hesperidin and chitooligosaccharide. They reported that obtained product has stronger antioxidant properties than hesperidin [9]. The antioxidant properties of the flavonoid rutin and two derivatives: rutin laurate and rutin palmitate were investigated showing decreased reducing power and metal chelating abilities as compared to rutin [10]. The use of the colloidal lipid formulae loaded with rutin as dermal care products was studied by Kamel and Mostafa [11]. The reported colloidal system of rutin was employed as a successful photoprotective product. Baldisserotto et al. present the synthesis, antimicrobial, antiproliferative and pro-apoptotic effect on human leukemic $\mathrm{K} 562$ cells of synthesized rutin derivatives [12]. The newly synthesized transglycosylated compound

$\triangle$ Iwona Binkowska, iwonakal@amu.edu.pl| 'Faculty of Chemistry, Adam Mickiewicz University, Uniwersytetu Poznańskiego 8 , 61-614 Poznan, Poland. 
from flavonoid, rhoifolin was reported by Aoki [13]. The encapsulation of rutin [14-16] and quercetin [17] was proposed as a way to obtain a product with protective effects against oxidative stress. Silica-based materials are suitable for oral delivery of drugs [18-22]. The advantage of silica is the simplicity of chemical modification due the presence of hydroxyl on the inner and outer surface of the material. The complexes of hesperidin may improve its physicochemical and pharmacological properties. Therefore, the objective of this work was to investigate the ability of hesperidin to form complexes with aminofunctionalized silica, characterized by thermogravimetric, spectrophotometric method, electron scanning microscopy and zeta potential. Herein, the derivatives of hesperidin were assessed for their antioxidant properties, and compared with their parent compound, hesperidin.

\section{Experimental}

\subsection{Chemicals}

Hesperidin (Hesperetin 7-rhamnoglucoside, Hesperitin-7-rutinoside, Empirical Formula $\mathrm{C}_{28} \mathrm{H}_{34} \mathrm{O}_{15}$, Molecular Weight: 610.56) was purchased from Sigma-Aldrich Co. (USA) and used without further purification. Silica: Syloid ${ }^{\circledR} 72$ was obtained from Grace Davison (USA)—synthetic and amorphous silica with average particle size: 2.5-12 microns. Silica was dried in $100^{\circ} \mathrm{C}$ overnight before modification. (3-aminopropyl)trimethoxysilane-APTMS was purchased from Degussa (Germany). Methanol (pure for analysis, POCH, Poland) was used in all experiments.

\subsection{Modification of silica and preparation of complex of modified silica with hesperidin}

The modified silica was prepared by reaction of Syloid ${ }^{\circledR} 72$ with (3-aminopropyl)trimethoxysilane. Various quantities of (3-aminopropyl)trimethoxysilane (0.1, 0.2 and $0.3 \mathrm{ml}$ ) was added drop wise to suspension of $0.5 \mathrm{~g}$ of silica in $60 \mathrm{ml}$ of dry toluene. Then, the mixture was heated under reflux for $8 \mathrm{~h}$. The products were filtered and washed several times with toluene and methanol. Then, the samples labeled as AMS-1, AMS-2 and AMS- 3 was dried in $100^{\circ} \mathrm{C}$ overnight. The complex of aminofunctionalized silica and hesperidin was prepared using various hesperidin/silica ratios $-1 / 1,1 / 2$ and $2 / 1$. The calculated amount of hesperidin was dissolved in methanol and then the modified silica was added. The mixture was stirred on magnetic stirrer in room temperature for $24 \mathrm{~h}$. Afterwards, the samples were filtered and washed with methanol. The samples labeled as: HESP-AMS-1, HESP-AMS-2 and HESP-AMS-3 were dried in $100^{\circ} \mathrm{C}$ for $12 \mathrm{~h}$. The percentage of hesperidin linked with aminofunctionalized silica was calculated from spectrophotometric and thermogravimetric analysis.

\subsection{The zeta potential}

The zeta potential was determined with Metasizer 3000 (0.6-1000 nm) instrument (Malvern Instruments Ltd., UK) using the non-invasive dynamic light scattering method (DLS).

\subsection{SEM images}

The morphological characterization of products was performed by electron scanning microscopy (Zeiss EVO40, Germany). The samples were previously coated with gold.

\subsection{FT-IR analysis}

Chemical properties of samples were analyzed as $\mathrm{KBr}$ pellets using a Bruker IFS 66v/S instrument. Spectral scanning was taken in the wavelength region between 400 and $4000 \mathrm{~cm}^{-1}$ with a resolution of $2 \mathrm{~cm}^{-1}$. 64 scans were taken with the average from each spectrum. The background of pure $\mathrm{KBr}$ spectrum was subtracted from the sample spectrum.

\subsection{TG analysis}

Thermal stability analysis of samples was performed by Setsys 1200 (Setaram, France) instrument. Obtained materials were analyzed under helium in the range of temperature between 16 and $1000^{\circ} \mathrm{C}$ and $5^{\circ} \mathrm{C} / \mathrm{min}$.

\subsection{Total antioxidant scavenging activity}

2,2-Diphenyl-2-picrylhydrazyl (DPPH) assay [23, 24] were chosen to investigate the free radical scavenging activity. The method is based on the observation of the changes in optical density of DPPH radicals. First, 2,2-diphenyl-1-picrylhydrazyl radical (DPPH) was dissolved in methanol to obtain $5 \times 10^{-5} \mathrm{M}$ solution. Then, the stock solution was then diluted to obtain a final absorbance about 1.0. The solutions of pure hesperidin labeled as: HESP-1, HESP-2 and HESP- 3 contain 1, 3 and $7 \mathrm{mg}$ of free hesperidin, respectively. The samples of hesperidin complex were suspended in methanol. Then, the solution of DPPH was added and the samples were stored for $30 \mathrm{~min}$ at room temperature in the dark. The absorbance was measured at $516 \mathrm{~nm}$ using a UV-visible spectrophotometer and methanol was used as a blank. Values are expressed as mean of three measurements. The free radical scavenging activity are calculated as percentage of inhibition (IC\%), according to formula: IC\% $=\left[\left(\mathrm{Abs}_{\mathrm{DPPH}}-\mathrm{Abs}_{\text {sample }}\right) / \mathrm{Abs}_{\mathrm{DPPH}}\right] \times 100 \%$, 
where: $\mathrm{Abs}_{\mathrm{DPPH}}$ and $\mathrm{Abs}_{\text {sample }}$ correspond to the absorbance of DPPH solution and antioxidant sample, respectively $[23,24]$. Trolox was used as a standard and results are expressed as $\mathrm{mg}$ trolox equivalents $\left(\mathrm{T}_{\mathrm{eq}}\right)$.

\subsection{Spectrophotometric measurements}

The absorbance was determined from electronic spectra recorded in methanol on a Hewlett Packard Diode Array Spectrophotometer HP 8452 fitted with thermostated cell holder to keep the temperature constant within $\pm 0.1^{\circ} \mathrm{C}$. All measurements are performed in triplicate. Results are expressed as mean values with standard deviation.

\subsection{Color analysis}

The spectral reflectance and CIELab coordinates of the studied complexes were measured using UV-Vis spectrophotometer Jasco V-630 (Japan) equipped with illuminant D65 and observer placed at $2^{\circ}$. The spectral range of the apparatus was $300-1000 \mathrm{~nm}$. Sample placed in a spherical cuvette (the $20 \mathrm{~mm}$ diameter) and a calibration with white standard-pure $\mathrm{BaSO}_{4}$.

\section{Results and discussion}

The aim of this research was to characterize the complex formed by hesperidin and aminofunctionalized silica. The studied complexes are synthesized according method described in experimental section. The physical and chemical characterization was performed using different techniques. First of all, the efficiency of reaction between hesperidin and functionalized silica was confirmed by spectrophotometric and thermogravimetric method. The results collected in Table 1 indicate that the efficiency of hesperidin complexation increases with increasing hesperidin/silica ratio. However, the duplication of the amount of hesperidin does not significantly improve the obtained results. Thermogravimetric analysis showed the content of a small, 1.0-1.2\% water content in the studied samples. The results indicate good compatibility of data obtained with spectrophotometric and thermogravimetric analysis.

The physical stability is a required condition essential for the characterization of the obtained product, being quantified by the evaluation of the zeta potential, defined as a parameter that describes the electric charge on the particles surface [25-28]. In aqueous solution the zeta potential depends on the $\mathrm{pH}$ value. The Fig. 1 shows the changes of zeta potential as a function of $\mathrm{pH}$. The results indicate that the unmodified silica has negative zeta potential through the $\mathrm{pH}$ range that was analyzed and is stable in the basic medium what is affected by presence of the deprotonated silanol groups. After modification process by aminosilan, the zeta potential increased significantly (Fig. 1). This effect is caused by the introduction of the amino groups on the silica surface. In the acidic medium, the amino groups are protonated which generated the positive charge and increase the zeta potential. The isoelectric point of unmodified silica is determined to be approximately 4.0 and differ considerably after silica modification.

The Fig. 1 show that the isoelectric point of modified silica is found at $\mathrm{pH}$ 9.68. The experimental $\mathrm{pH}$ range of stability shifts to the lower values of $\mathrm{pH}$ scale. The attaching a hesperidin to modified silica does not change significantly the stability of resulted molecules in comparison with functionalized silica. The isoelectric point was shifted after reaction of about one $\mathrm{pH}$ unit, from $\mathrm{pH} 9.68$ to $\mathrm{pH} 8.55$ and the values of zeta potential decreased slightly. This result is caused by a reduction of number of free amino groups after reaction and presence of hydroxyl groups of hesperidin. It is also annotate that at $\mathrm{pH}$ values higher than isoelectric point, the negative values of zeta potential were determined for AMS (around $-4 \mathrm{mV}$ at $\mathrm{pH} 10$ ) and AMS-HESP (-26 mV), which results from the deprotonation of silanol groups of silica surface.

The characterization of described complex between hesperidin and aminofunctionalized silica has been based on the information obtained with different techniques. The thermogravimetric analysis provided information on the amount of hesperidin linked with silica as well as the thermal stability of obtained complex.
Table 1 The results of thermogravimetric and spectrophotometric analysis

\begin{tabular}{llccc}
\hline Sample & $\begin{array}{l}\text { HESP/AMS } \\
\text { ratio }\end{array}$ & \% hesperidin, TG & \% hesperidin, UV-vis & $\begin{array}{l}\text { \% of } \\
\text { adsorbed } \\
\text { water }\end{array}$ \\
\hline HESP-AMS-1 & $1 / 2$ & $12.5 \pm 0.3$ & $15.0 \pm 0.1$ & $1.1 \pm 0.1$ \\
HESP-AMS-2 & $1 / 1$ & $15.0 \pm 0.4$ & $16.2 \pm 0.2$ & $1.2 \pm 0.1$ \\
HESP-AMS-3 & $2 / 1$ & $15.2 \pm 0.3$ & $16.1 \pm 0.2$ & $1.0 \pm 0.2$ \\
AMS & - & - & - & $1.1 \pm 0.2$ \\
\hline
\end{tabular}


Fig. 1 Zeta potential of: (a) unmodified silica, (b) aminofunctionalized silica and (c) complex of hesperidin with aminofunctionalized silica as a function of $\mathrm{pH}$

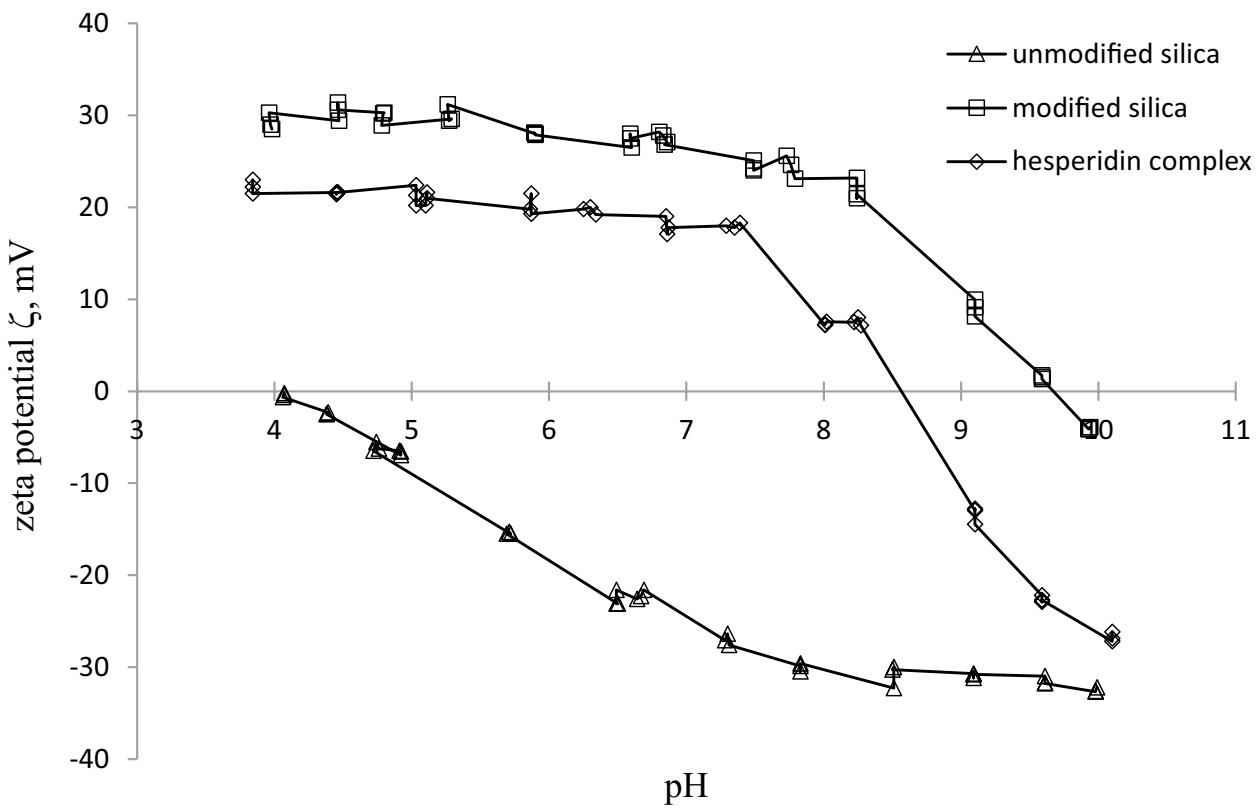

The thermal decomposition profiles obtained for modified silica, hesperidin and hesperidin complex, acquired in the same experimental conditions are showed in Fig. 2. Comparison of TG curves for modified silica and complex with hesperidin shows the high thermal stability of the studied complex. The complexation reaction with hesperidin affects the thermal stability of modified silica. The results indicate that in a first part of TG curves of AMS and AMS-HESP complex a 1-1.2\% weight loss was observed what is associated with the loss of surface water. In the next step, the thermal decomposition of samples is observed. Between 100 and $250^{\circ} \mathrm{C}$, the curves are almost flat suggesting high stability of material in this temperature range. The decomposition of AMS and AMS-HESP complex occurs above $300^{\circ} \mathrm{C}$. The Fig. 2 shows also the thermal stability of obtained AMS-HESP complex with a $12.5-15.2 \%$ loss of weight (Table1). However, the pure hesperidin has low thermal stability, reported previously by Cao et al. They described the loss of weight about $60 \%$ between 266 and $500{ }^{\circ} \mathrm{C}$ [9]. The results obtained for pure hesperidin are similar to those in literature (Fig. 2).

The obtained samples were also characterized morphologically by electron scanning microscopy. The selected SEM images are presented in Fig. 3. The unmodified silica and the modified silica form undefined, irregular flakeshaped particles. For comparison, the crystals of free
Fig. 2 Thermogravimetric analysis of amino modified silica, hesperidin and complex of hesperidin with aminofunctionalized silica

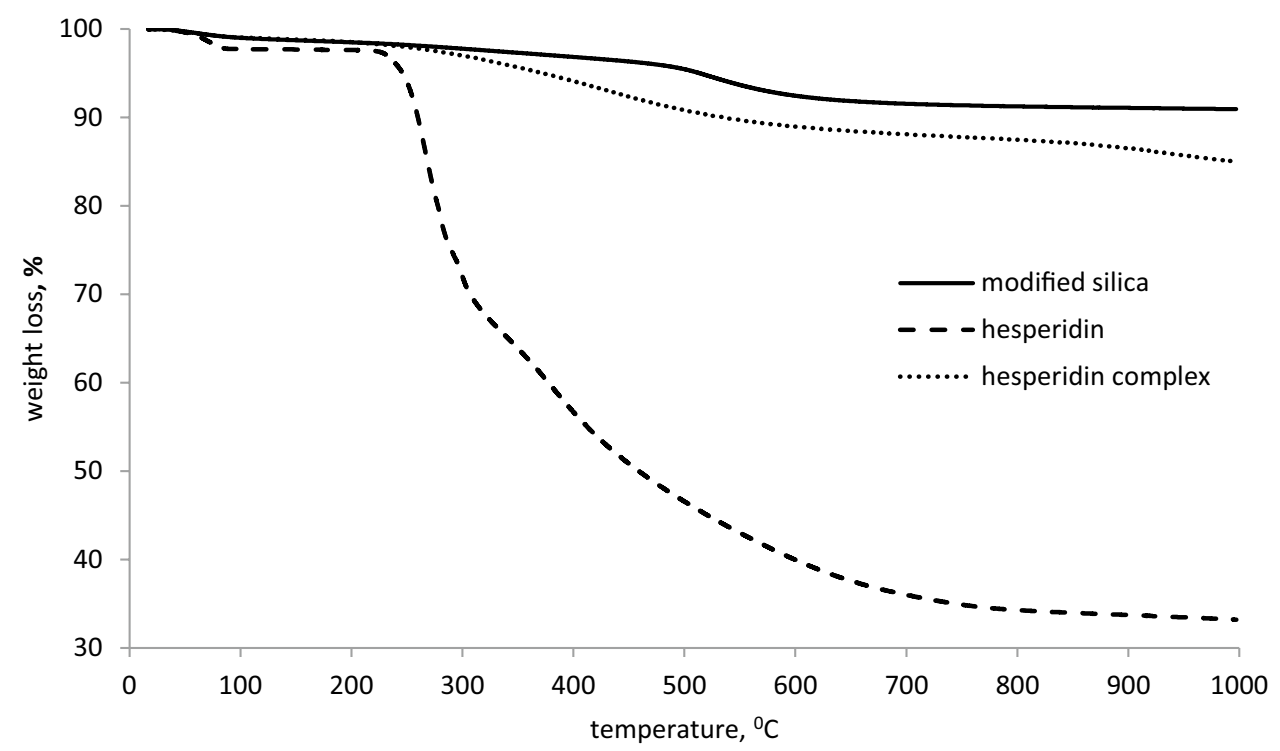



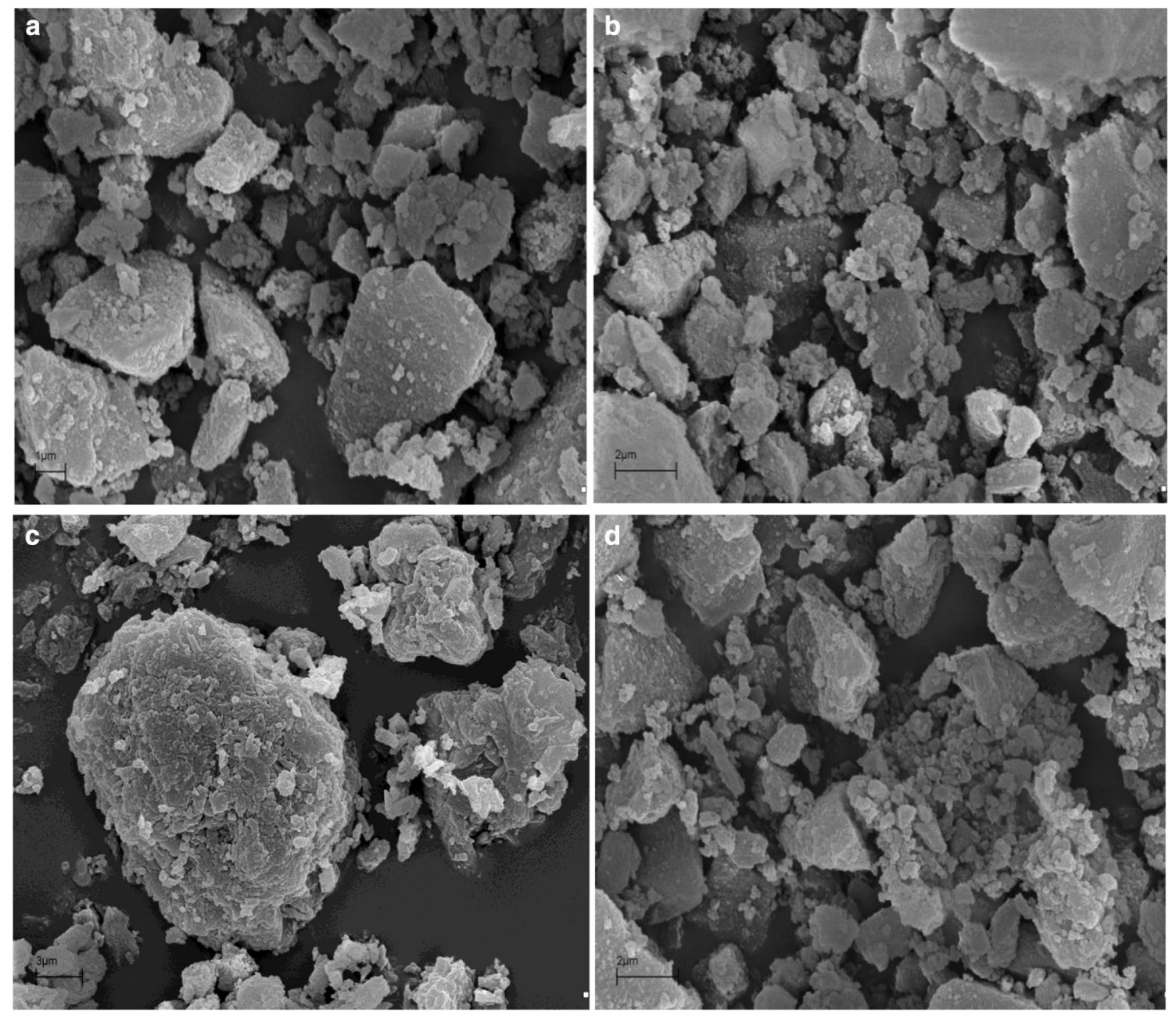

Fig. 3 Scanning electron microscopy images: a unmodified silica, b aminofunctionalized silica, $\mathbf{c}$ hesperidin, $\mathbf{d}$ hesperidin-modified silica complex

hesperidin and its complex with amino modified silica are showed. The morphology of functionalized silica is no different from the starting material. The association of amino functionalized silica with hesperidin also does not change significantly the shape of obtained particles. The reaction products appeared in the form of irregular particles. Besides the color, the hesperidin complex does not differ in its appearance from the starting silica. The results of the studies described in the paper confirm that the reaction took place and the expected product was obtained. The color of the samples, whitish for silica and yellow for the hesperidin complex is a clear difference in appearance. The color analysis presented later in this paper confirm these results.

FT-IR spectra of the hesperidin complex and pure substance were compared. The effect of silica modification is confirmed by the differences in FT-IR spectra. The intensity of the band at $3200-3600 \mathrm{~cm}^{-1}$, assigned for the $\mathrm{OH}$ stretching vibrations of silanol groups decreases in comparison with the band in the spectrum of the unmodified silica. Next, the intensity of the band at $3005-2930 \mathrm{~cm}^{-1}$ assigned to $\mathrm{C}-\mathrm{H}$ increases after amino modification, which is connected with presence of alkyl groups. The intensity of this band increases significantly after modification of silica and shifted from 2987 to $2949 \mathrm{~cm}^{-1}$. The band at $1560 \mathrm{~cm}^{-1}$ corresponds to the $\mathrm{N}-\mathrm{H}$ stretching vibration confirmed the amino modification of silica. Moreover, the changes are also observed at region $800-1000 \mathrm{~cm}^{-1}$ assigned to $\mathrm{Si}-\mathrm{OH}$ vibration. The intensity of the band visible at $988 \mathrm{~cm}^{-1}$ decreases after modification of silica. Furthermore, in spectra of unmodified and modified silica, the characteristic broad and intense band is observed at around $1100 \mathrm{~cm}^{-1}$ due to $\mathrm{Si}-\mathrm{O}-\mathrm{Si}$ vibrations coming from silica matrix. The bands corresponding to the $\mathrm{Si}-\mathrm{O}$ vibrations appear at 801 and $475 \mathrm{~cm}^{-1}$.

The changes in the characteristic bands of pure hesperidin and modified silica confirm the formation of the reaction product. In the spectrum of free hesperidin 
(Fig. 4), characteristic large absorption bands assigned to $\mathrm{OH}$ stretching vibrations at 3448 and $3552 \mathrm{~cm}^{-1}$ were present. The other band at $2940 \mathrm{~cm}^{-1}$ of $\mathrm{CH}$ and $\mathrm{CH}_{2}$ aliphatic groups is observed. The next sharp bands at 1650 and $1638 \mathrm{~cm}^{-1}$ are specific for the carbonyl group and aromatic $C=C$ vibration in benzene ring. After the reaction between free hesperidin and aminofunctionalized silica a complex is formed as showed in the FT-IR spectrum. The intensity of the bands for $\mathrm{OH}$ vibrations, aliphatic and carbonyl groups are visibly decreased suggesting the interaction between the hesperidin molecule and surface of the amino modified silica.

In order to investigate or the antioxidant properties of hesperidin will also be observed in prepared complexes, antioxidant tests for the complex and pure hesperidin were performed. For the DPPH free radical scavenging assay at different concentrations of hesperidin and complexes were used. The experimental method is based on the reduction of DPPH radical by hesperidin which contains hydroxyl groups.

Table 2 and Fig. 5 show the free radical scavenging activity determined for studied complex with aminofunctionalized silica. For comparison, an analogous experiment with free hesperidin was carried out. Results show that complex of hesperidin with modified silica exhibits antioxidant properties, slightly higher than in the case of the parent compound. The next stage of the research was to check the dependence of the obtained result on the hesperidin amount (1, 3 and $7 \mathrm{mg}$ of hesperidin). For this purpose, absorbance measurements for three solutions of free hesperidin as well as studied complexes were taken. An increase in scavenger capacity with increasing concentration of free hesperidin was observed (Fig. 5).
Table 2 The total scavenging activity of hesperidin-silica complexes in comparison with pure hesperidin: a percentage of inhibition (IC) and trolox equivalent antioxidant capacity $\left(\mathrm{T}_{\text {eq }}\right)$

\begin{tabular}{|c|c|c|c|}
\hline Sample & $\Delta \mathrm{Abs} \pm \mathrm{SD}$ & IC (\%) & $\mathrm{T}_{\text {eq }}(\mu \mathrm{M})$ \\
\hline \multicolumn{4}{|c|}{ Functionalized silica, AMS } \\
\hline & $0.024 \pm 0.001$ & $2.3 \pm 0.1$ & $0.7 \pm 0.1$ \\
\hline \multicolumn{4}{|l|}{ Free hesperidin } \\
\hline HESP-1 & $0.513 \pm 0.001$ & $50.8 \pm 0.1$ & $13.2 \pm 0.1$ \\
\hline HESP-2 & $0.656 \pm 0.001$ & $60.3 \pm 0.2$ & $15.5 \pm 0.1$ \\
\hline HESP-3 & $0.763 \pm 0.001$ & $75.5 \pm 0.2$ & $17.8 \pm 0.1$ \\
\hline \multicolumn{4}{|c|}{ Hesperidin-AMS complex } \\
\hline HESP-AMS-1 & $0.603 \pm 0.001$ & $59.7 \pm 0.1$ & $14.8 \pm 0.1$ \\
\hline HESP-AMS-2 & $0.784 \pm 0.001$ & $60.3 \pm 0.1$ & $15.4 \pm 0.1$ \\
\hline HESP-AMS-3 & $0.908 \pm 0.002$ & $75.5 \pm 0.2$ & $16.5 \pm 0.1$ \\
\hline
\end{tabular}

$\Delta \mathrm{Abs}=\mathrm{A}_{0}-\mathrm{A}$, where $\mathrm{A}_{0}$-initial absorbance at $516 \mathrm{~nm}, \mathrm{~A}$-absorbance at $516 \mathrm{~nm}$ after reaction

Further, it was found that the antioxidant capacity change with increasing concentration of hesperidin in complex. For comparison, the value of percentage of inhibition for modified silica is shown, indicating weak antioxidant properties (Table 2, Fig. 5).

Figure 6 shows the reflectance spectrum of the hesperidin-silica complexes for the spectral range of $300-1000 \mathrm{~nm}$. It can be seen that the reflectance spectrum increases gradually from $450 \mathrm{~nm}$. The reflectance of complexes changes with different amounts of hesperidin. Low content of hesperidin results in higher reflectance and vice versa. For comparison, the reflectance spectrum for modified silica (AMS) is given.

CIELab values of studied complexes are given in Table 3 . It can be seen that the amount of hesperidin has an
Fig. 4 FT-IR spectra of unmodified silica, modified silica, free hesperidin and hesperidin complex

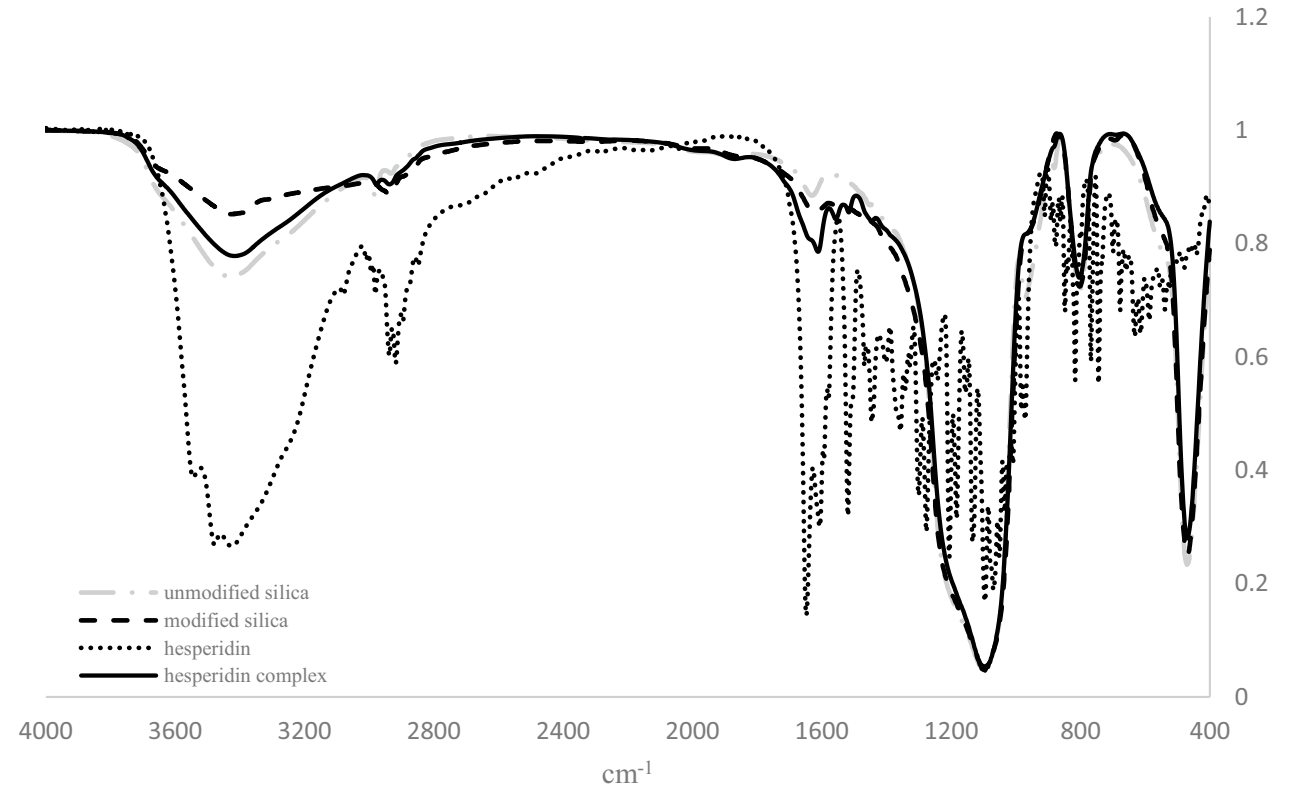

1.2

.8

0.6

$\mathrm{cm}^{-1}$ 
Fig. 5 Percentage reductions in the absorbance of 2,2-diphenyl-2-picrylhydrazyl solution (\% IC) in the presence of different amounts of free hesperidin and hesperidin complex. For comparison, \% IC is given for the modified silica

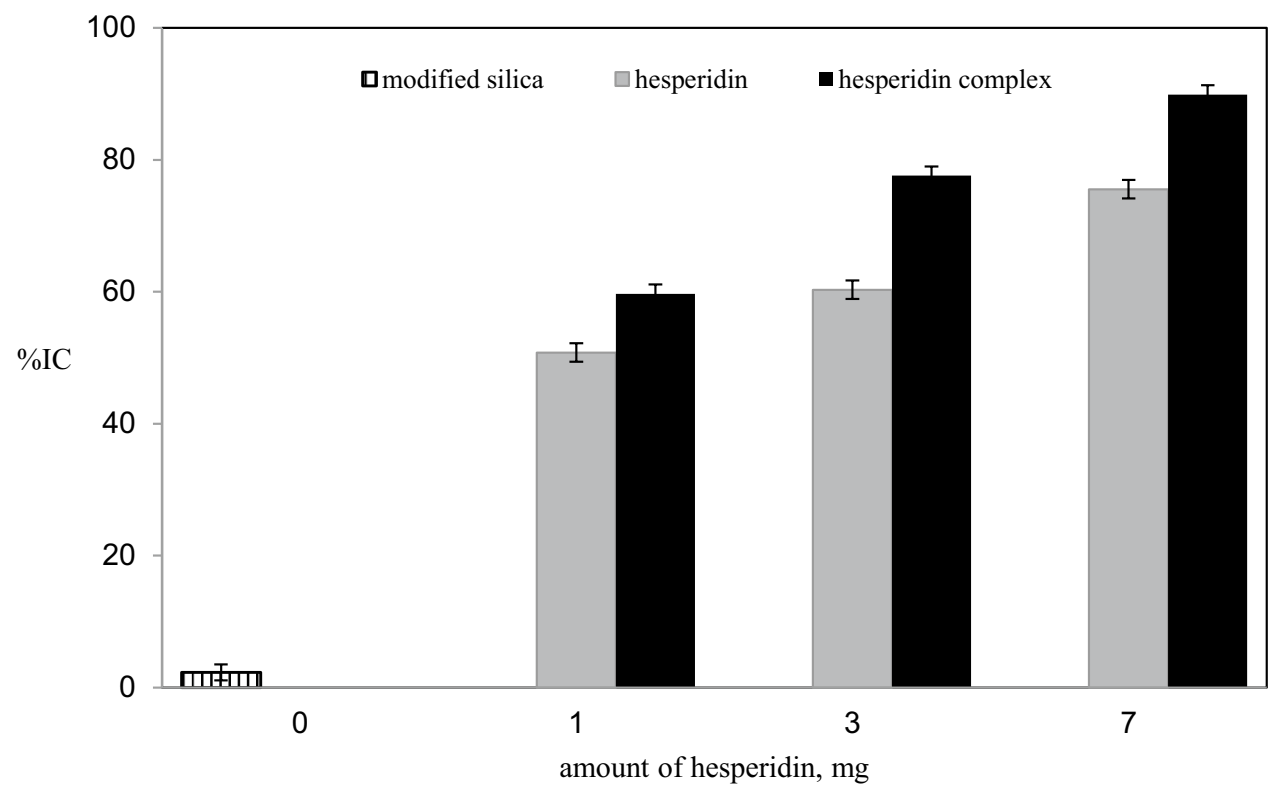

Fig. 6 Reflectance spectra for hesperidin complexes (HESP-1, HESP-2, HESP-3) and modified silica (AMS)

Table 3 Color analysis of unmodified, modified (AMS) silica free hesperidin (HESP) and hesperidin complexes (HESP-1, HESP-2, HESP-3)

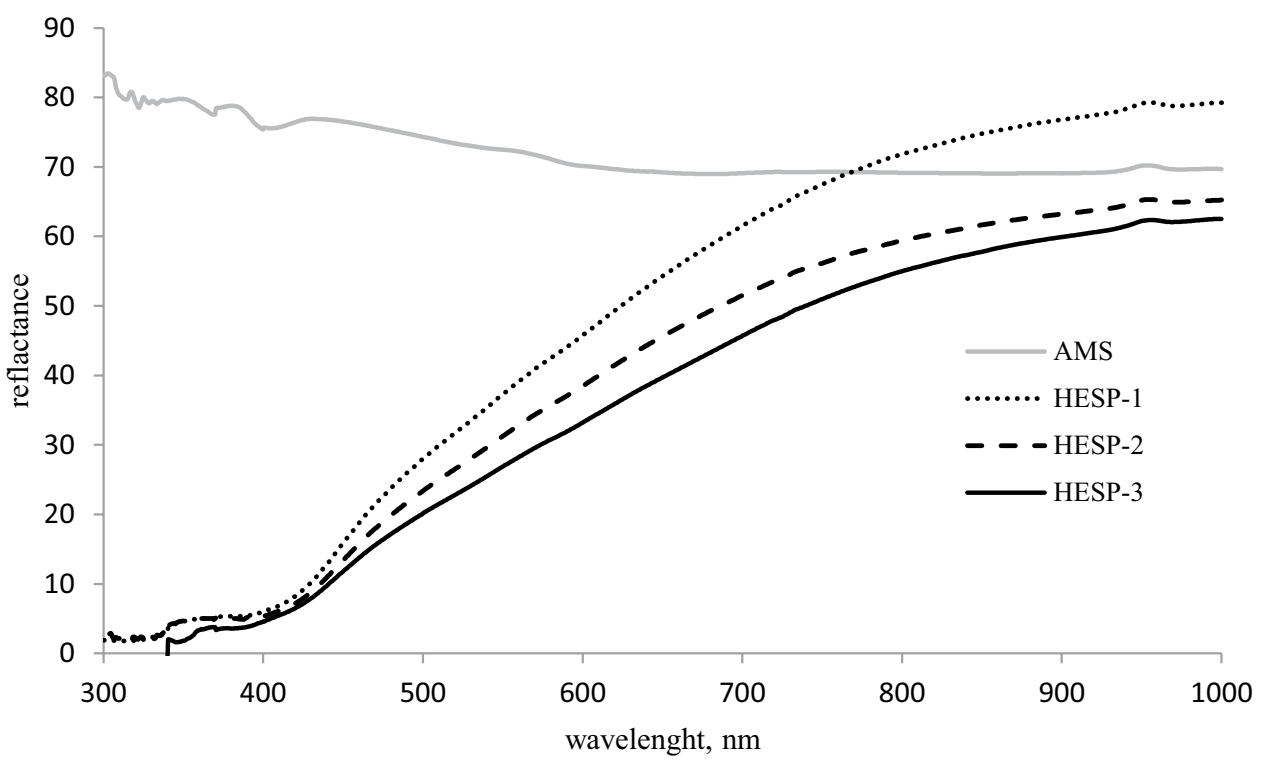

\begin{tabular}{llrrrr}
\hline Sample & \multicolumn{1}{l}{$\mathrm{L}^{*}$} & \multicolumn{1}{l}{$\mathrm{a}^{*}$} & \multicolumn{1}{l}{$\mathrm{b}^{*}$} & \multicolumn{1}{l}{ Chroma } & \multicolumn{1}{c}{ Hue $\left({ }^{\circ}\right)$} \\
\hline Unmodified silica & $87.9 \pm 0.2$ & $-0.6 \pm 0.1$ & $-0.3 \pm 0.2$ & $1.2 \pm 0.1$ & $-3.0 \pm 0.2$ \\
AMS & $85.9 \pm 0.2$ & $-0.5 \pm 0.1$ & $-4.2 \pm 0.2$ & $4.5 \pm 0.2$ & $-2.6 \pm 0.2$ \\
HESP & $72.4 \pm 0.1$ & $-1.1 \pm 0.1$ & $17.3 \pm 0.1$ & $17.3 \pm 0.1$ & $-86.3 \pm 0.2$ \\
HESP-1 & $68.5 \pm 0.2$ & $3.4 \pm 0.1$ & $33.4 \pm 0.1$ & $33.5 \pm 0.2$ & $81.2 \pm 0.2$ \\
HESP-2 & $63.5 \pm 0.3$ & $3.6 \pm 0.1$ & $32.6 \pm 0.1$ & $31.8 \pm 0.1$ & $83.6 \pm 0.2$ \\
HESP-3 & $59.7 \pm 0.3$ & $3.8 \pm 0.2$ & $29.5 \pm 0.1$ & $29.8 \pm 0.2$ & $83.8 \pm 0.1$
\end{tabular}

Chroma $=\left[\left(a^{*}\right) 2+\left(b^{*}\right) 2\right] 1 / 2$, Hue angle $=\tan ^{-1}\left(b^{*} / a^{*}\right)$ important effect on colorimetric properties. It is known that $L^{*}$ indicates the specific brightness of the pigment from 0 (black) to 100 (white). Values of $b^{*}$ greater than 0 represent yellow color. With these values, the values of hue angle $\left(\mathrm{Hue}^{\circ}\right)$ and Chroma or saturation of the pigment were calculated. Hue angle value denotes 0 for redness, 90 
for yellowness, 180 for greenness, and 270 for blueness. The Chroma of hesperidin-silica complexes was from 29.8 to 33.5 and the Hue angle from 81.2 to 83.6 , which represents colors in the yellow region. It is consistent with the literature data. In the study evaluating the color of yellow pigments, an Hue angle of 84.37 was obtained, similar to that observed in this work [29]. Other investigation described by Venil et al. [30] show that the Hue angle for yellow pigment isolated from Chryseobacterium was 77.39. Lightness value $\left(L^{*}\right)$ of hesperidin-silica complexes was found to be inversely correlated with the antioxidant activity values with the correlation coefficient of 0.994 . This value could be a good indicator for the estimate of these products.

\section{Conclusion}

In this study, the method for preparation of complex between hesperidin and functionalized silica was developed. Presented investigations could play an important role to improvement the reported properties of hesperidin. The obtained material has been characterized and the antioxidant scavenging activity was evaluated. The analytical methods used, such as TG, UV-vis, FT-IR and potential zeta, show that the reaction took place and the expected product was obtained, although there are no noticeable morphological differences in the appearance of these samples. The color of the samples, whitish for silica and yellow for the hesperidin complex is a clear difference in appearance. The results of the CIELab color analysis and the reflectance spectra confirm these differences. The presented results lead to the following conclusions:

- The proposed method has facility to design the simple production of material with preferred properties

- The hesperidin complex has good stability as it appears from the zeta potential measurements

- The antioxidant properties of obtained complex has enhanced in comparison with free hesperidin

Funding This study received financial support from the Polish Ministry of Science and Higher Education for the Faculty of Chemistry of Adam Mickiewicz University in Poznan under statutory activity subsidies.

\section{Compliance with ethical standards}

Conflict of interest The authors report no conflict of interest in this work.
Open Access This article is licensed under a Creative Commons Attribution 4.0 International License, which permits use, sharing, adaptation, distribution and reproduction in any medium or format, as long as you give appropriate credit to the original author(s) and the source, provide a link to the Creative Commons licence, and indicate if changes were made. The images or other third party material in this article are included in the article's Creative Commons licence, unless indicated otherwise in a credit line to the material. If material is not included in the article's Creative Commons licence and your intended use is not permitted by statutory regulation or exceeds the permitted use, you will need to obtain permission directly from the copyright holder. To view a copy of this licence, visit http://creativecommons .org/licenses/by/4.0/.

\section{References}

1. Puri M, Verma ML, Mahale K (2012) Processing of citrus peel for the extraction of flavonoids for biotechnological applications. In: Yamane K, Kato Y (eds) Handbook on flavonoids. Nova Science Publishers, Inc., Hauppauge

2. Yao LH, Jiang YM, Shi J, Tomas-Barber FA, Datta N, Singanusong $R$, Chen SS (2004) Flavonoids in food and their health benefits. Plant Foods Hum Nutr 59:113-122

3. Roohbakhsh A, Parhiz H, Soltani F, Rezaeea R, Iranshahi M (2015) Molecular mechanisms behind the biological effects of hesperidin and 3 hesperetin for the prevention of cancer and cardiovascular disease. Life Sci 124:64-74. https://doi.org/10.1016/j. Ifs.2014.12.030

4. Agati G, Azzarello E, Pollastri S, Tattini M (2012) Flavonoids as antioxidants in plants: location and functional significance. Plant Sci 196:67-76. https://doi.org/10.1016/j.plantsci.2012.07.014

5. Ahmadi A, Shadboorestan A (2016) Oxidative stress and cancer; the role of hesperidin, a citrus natural bioflavonoid, as a cancer chemoprotective agent. Nutr Cancer 68:29-39. https://doi. org/10.1080/01635581.2015.1078822

6. Roohbakhsh A, Parhiz H, Soltani F, RezaeeaR IM (2014) Neuropharmacological properties and pharmacokinetics of the citrus flavonoids hesperidin and hesperetin-a mini-review. Life Sci 113:1-6. https://doi.org/10.1016/j.lfs.2014.07.029

7. Sahu N, Soni D, Chandrashekhar B, Satpute DB, Saravanadevi S, Sarangi BK, Pandey RA (2016) Synthesis of silver nanoparticles using flavonoids: hesperidin, naringin and diosmin, and their antibacterial effects and cytotoxicity. Int Nano Lett 6:173-181. https://doi.org/10.1007/s40089-016-0184-9

8. Tan M, Zhu J, Pan Y, Chen Z, Liang H, Liu H, Wang H (2009) Synthesis, cytotoxic activity, and DNA binding properties of copper(II) complexes with hesperetin, naringenin, and apigenin. Bioinorg Chem Appl 2009:1-9. https://doi.org/10.1155/2009/347872

9. Cao R, Zhao Y, Zhou Z, Zhao X (2018) Enhancement of the water solubility and antioxidant activity of hesperidin by chitooligosaccharide. J Sci Food Agric 98:2422-2427. https://doi. org/10.1002/jsfa.8734

10. Lue BM, Nielsen NS, Jacobsen C, Hellgren L, Guo Z, Xu X (2010) Antioxidant properties of modified rutin esters by $\mathrm{DPPH}$, reducing power, iron chelation and human low density lipoprotein assays. Food Chem 123:221-230. https://doi.org/10.1016/j.foodc hem.2010.04.009

11. Kamel R, Mostafa DM (2015) Rutin nanostructured lipid cosmeceutical preparation with sun protective potential. J Photochem Photobiol B Biol 153:59-66. https://doi.org/10.1016/j.jphotobiol .2015.09.002

12. Baldisserotto A, Vertuani S, Bino A, Lucia DD, Lampronti I, Milani R, Gambari R, Manfredini S (2015) Design, synthesis and biological activity of a novel Rutin analogue with improved lipid 
soluble properties. Bioorg Med Chem 23:264-271. https://doi. org/10.1016/j.bmc.2014.10.023

13. Aoki C, Takeuchi Y, Higashi K, Okamoto Y, Nakanishi A, Tandia M, Uzaw J, Ueda K, Moribe K (2017) Structural elucidation of a novel transglycosylated compound a-glucosyl rhoifolin and of a-glucosyl rutin by NMR spectroscopy. Carbohydr Res 443444:37-41. https://doi.org/10.1016/j.carres.2017.03.011

14. Bonechi C, Donati A, Tamasi G, Leone G, Consumi M, Rossi C, Lamponi S, Magnani A (2018) Protective effect of quercetin and rutin encapsulated liposomes on induced oxidative stress. Biophys Chem 233:55-63. https://doi.org/10.1016/j. bpc.2017.11.003

15. Yang R, Liu Y, Blanchard Ch, Zhou Zh (2018) Channel directed rutin nano-encapsulation in phytoferritin induced by guanidine hydrochloride. Food Chem 240:935-939. https://doi. org/10.1016/j.foodchem.2017.07.088

16. Wei Q, Keck CM, Müller RH (2017) Preparation and tableting of long-term stable amorphous rutin using porous silica. Eur J Pharm Biopharm 113:97-107. https://doi.org/10.1016/j. ejpb.2016.11.009

17. Diamantis DA, Ramesova S, Chatzigiannis M, Degano I, Gerogianni S, Karadima KE, Perikleous S, Rekkas D, Gerothanassis IP, Galaris D, Mavromoustakos T, Valsami G, Sokolova R, Tzakos AG (2018) Exploring the oxidation and iron binding profile of a cyclodextrin encapsulated quercetin complex unveiled a controlled complex dissociation through a chemical stimulus. BBA Gen Subj 1862:1913-1924. https://doi.org/10.1016/j.bbage n.2018.06.006

18. Diab R, Canilho N, Pavel IA, Haffner FB, Girardon M, Pasc A (2017) Silica-based systems for oral delivery of drugs, macromolecules and cells. Adv Colloid Int Sci 249:349-362. https:// doi.org/10.1016/j.cis.2017.04.005

19. Thaher YA, Perni S, Prokopovich P (2017) Nano-carrier based drug delivery systems for sustained antimicrobial agent release from orthopaedic cementous material. Adv Colloid Int Sci 249:234-247. https://doi.org/10.1016/j.cis.2017.04.017

20. Finnie KS, Waller DJ, Perret FL, Krause-Heuer AM, Lin HQ, Hanna JV, Barbe ChJ (2009) Biodegradability of sol-gel silica microparticles for drug delivery. J Sol-Gel Sci Technol 49:12-18. https:// doi.org/10.1007/s10971-008-1847-4

21. Tan L, Wu T, Tang ZhW, Xiao JY, Zhuo RX, Shi B, Liu ChJ (2016) Water-soluble photoluminescent fullerene capped mesoporous silica for $\mathrm{pH}$ responsive drug delivery and bioimaging. Nanotechnology 27:315104-315114. https://doi.org/10.1088/0957$4484 / 27 / 31 / 315104$
22. Mendez-Gonzalez D, Lopez-Cabarcos E, Rubio-Retama LJM (2017) Sensors and bioassays powered by upconverting materials. Adv Colloid Int Sci 249:66-87. https://doi.org/10.1016/j. cis.2017.06.003

23. Brand-Williams W, Cuvelier C, Berset ME (1995) Use of a free radical method to evaluate antioxidant activity. LWT Food Sci Technol 28:25-30. https://doi.org/10.1016/S0023-6438(95)80008-5

24. Yen GCh, Duh PD (1994) Scavenging effect of methanolic extracts of peanut hulls on free-radical and active-oxygen species. J Agric Food Chem 42:629-632. https://doi.org/10.1021/ jf00039a005

25. Bhhatacharjee $S$ (2016) DLS and zeta potential-What they are and what they are not? J Control Release 235:337-351. https:// doi.org/10.1016/j.jconrel.2016.06.017

26. Ch LW, SzH L, Karakachian MB, Yu Y, Chen YY, Lin ChY, Kuo ChH, Shyue JJ (2009) Tuning the surface potential of gold substrates arbitrarily with self-assembled monolayers with mixed functional groups. Phys Chem Chem Phys 11:6199-6204. https:// doi.org/10.1039/B902044F

27. Kuo ChH, Chang HY, Liu ChP, SzH L, You YW, Shyue JJ (2011) Effect of surface chemical composition on the surface potential and iso-electric point of silicon substrates modified with selfassembled monolayers. Phys Chem Chem Phys 13:3649-3653. https://doi.org/10.1039/COCP02615H

28. Arce VB, Gargarello RM, Ortega F, Romañano V, Mizrahi M, Ramallo-López JM, Cobosa CJ, Airoldi C, Bernardelli C, Donati ER, Mártire DO (2015) EXAFS and DFT study of the cadmium and lead adsorption on modified silica nanoparticles. Spectrochim Acta Part A Mol Biomol Spectr 151:156-163. https://doi. org/10.1016/j.saa.2015.06.093

29. Aruldass CA, Chidambaram AA, Ali KV, Wan RK, Ahmad A (2016) Utilization of agro-industrial waste for the production of yellowishorange pigment from Chryseobacterium artocarpi CECT 8497. Int Biodeterior Biodegrad 113:342-349. https://doi. org/10.1016/j.ibiod.2016.01.024

30. Venil ChK, Zakaria ZA, Usha R, Ahmad WA (2014) Isolation and characterization of flexirubin type pigment from Chryseobacterium sp. UTM-3T. Biocatal Agric Biotechnol 3:103-107. https ://doi.org/10.1016/j.bcab.2014.02.006

Publisher's Note Springer Nature remains neutral with regard to jurisdictional claims in published maps and institutional affiliations. 